1 Hacettepe Journal of Mathematics and Statistics

$\bigcap$ Volume 45 (3) (2016), 811-825

\title{
Chen inequalities for submanifolds of generalized space forms with a semi-symmetric metric connection
}

\author{
Sibel SULAR*
}

\begin{abstract}
We investigate sharp inequalities for submanifolds in both generalized complex space forms and generalized Sasakian space forms with a semisymmetric metric connection.
\end{abstract}

Keywords: Chen inequality, generalized complex space form, generalized Sasakian space form, semi-symmetric metric connection.

2000 AMS Classification: 53B05, 53B15, 53C40.

Received: 14.03.2012 Accepted: 11.04.2012 Doi : 10.15672/HJMS.20164517539

\section{Introduction}

A. Friedmann and J. A. Schouten introduced the idea of a semi-symmetric linear connection on a Riemannian manifold in [10]. Later, H. A. Hayden [11] gave the definition of a semi-symmetric metric connection. In 1970, K. Yano [19] studied semi-symmetric metric connection and proved that a Rimannian manifold admits a semi-symmetric metric connection with vanishing curvature tensor if and only if the manifold is conformally flat. Then, in [12], [13] and [16] T. Imai and Z. Nakao considered some properties of a Riemannian manifold admitting a semi-symmetric metric connection and they studied submanifolds of a Riemannian manifold with a semi-symmetric metric connection.

On the other hand, B. Y. Chen introduced Chen inequality and he gave the definition of new types of curvature invariants (called extrinsic and intrinsic invariants) in [6]. Then,

*Department of Primary Mathematics Education, Balikesir University, 10100, Balıkesir, TURKEY.

Email : csibel@balikesir.edu.tr 
in [7], [8] and [9], he established sharp inequalities for different submanifolds in various ambient spaces.

In [3] and [4], K. Arslan, R. Ezentaş, I. Mihai, C. Murathan and C. Özgür studied Chen inequalities for submanifolds in locally conformal almost cosymplectic manifolds and $(\kappa, \mu)$-contact space forms, respectively. Later, P. Alegre, A. Carriazo, Y. H. Kim and D. W. Yoon considered same inequalities for submanifolds of generalized space forms in [2].

Recently, in [14], A. Mihai and C. Özgür proved Chen inequalities for submanifolds of real space forms admitting a semi-symmetric metric connection. They also studied same problems for submanifolds of complex space forms and Sasakian space forms with a semi-symmetric metric connection in [15]. As a generalization of the results of [15], in tis study, we prove similar inequalities for submanifolds of generalized complex space forms and generalized Sasakian space forms with respect to a semi-symmetric metric connection.

\section{Preliminaries}

Let $N$ be an $(n+p)$-dimensional Riemannian manifold with a Riemannian metric $g$. A linear connection $\widetilde{\nabla}$ on a Riemannian manifold $N$ is called a semi-symmetric connection if the torsion tensor $\widetilde{T}$ of the connection $\widetilde{\nabla}$

$$
\widetilde{T}(\tilde{X}, \widetilde{Y})=\widetilde{\nabla}_{\widetilde{X}} \tilde{Y}-\widetilde{\nabla}_{\tilde{Y}} \tilde{X}-[\tilde{X}, \tilde{Y}]
$$

satisfies

$$
\widetilde{T}(\widetilde{X}, \widetilde{Y})=w(\widetilde{Y}) \widetilde{X}-w(\widetilde{X}) \widetilde{Y}
$$

for any vector fields $\widetilde{X}$ and $\tilde{Y}$ on $N$, where $w$ is a 1-form associated with the vector field $U$ on $N$ defined by

$$
w(\widetilde{X})=g(\widetilde{X}, U) .
$$

$\widetilde{\nabla}$ is called a semi-symmetric metric connection if

$$
\widetilde{\nabla} g=0 .
$$

If $\stackrel{\circ}{\widetilde{\nabla}}$ is the Levi-Civita connection of a Riemannian manifold $N$, the semi-symmetric metric connection $\widetilde{\nabla}$ is given by

$$
\widetilde{\nabla}_{\tilde{X}} \tilde{Y}=\stackrel{\circ}{\nabla}_{\widetilde{X}} \tilde{Y}+w(\tilde{Y}) \tilde{X}-g(\tilde{X}, \tilde{Y}) U
$$

(see [19]).

Let $M$ be an $n$-dimensional submanifold of an $(n+p)$-dimensional Riemannian manifold $N$. We will consider the induced semi-symmetric metric connection by $\nabla$ and the induced Levi-Civita connection by $\stackrel{\circ}{\nabla}$ on the submanifold $M$.

Let $\widetilde{R}$ and $\stackrel{\circ}{\widetilde{R}}$ be curvature tensors of $\widetilde{\nabla}$ and $\stackrel{\circ}{\widetilde{\nabla}}$ of a Riemannian manifold $N$, respectively. We also denote by $R$ the curvature tensor of $M$ with respect to $\nabla$ and $\stackrel{\circ}{R}$ the 
curvature tensor of $M$ with respect to $\stackrel{\circ}{\nabla}$. Then the Gauss formulas with a semi-symmetric metric connection $\nabla$ and the Levi-Civita connection $\stackrel{\circ}{\nabla}$, respectively, are given by

$$
\widetilde{\nabla}_{X} Y=\nabla_{X} Y+\sigma(X, Y)
$$

and

$$
\stackrel{\circ}{\nabla}_{X} Y=\stackrel{\circ}{\nabla}_{X} Y+\stackrel{\circ}{\sigma}(X, Y),
$$

for any vector fields $X, Y$ tangent to $M$, where $\stackrel{\circ}{\sigma}$ is the second fundamental form of $M$ in $N$ and $\sigma$ is a $(0,2)$-tensor on $M$. Also, the mean curvature vector of $M$ in $N$ is denoted by $\stackrel{\circ}{H}$.

The equation of Gauss for an $n$-dimensional submanifold $M$ in an $(n+p)$-dimensional Riemannian manifold $N$ is given by

$$
\stackrel{\circ}{\widetilde{R}}(X, Y, Z, W)=\stackrel{\circ}{R}(X, Y, Z, W)+g(\stackrel{\circ}{\sigma}(X, Z), \stackrel{\circ}{\sigma}(Y, W))-g(\stackrel{\circ}{\sigma}(Y, Z), \stackrel{\circ}{\sigma}(X, W))
$$

Then, $\widetilde{R}$ and $\stackrel{\circ}{R}$ are related by

$$
\begin{aligned}
\widetilde{R}(X, Y, Z, W)= & \stackrel{\circ}{\widetilde{R}}(X, Y, Z, W)-\alpha(Y, Z) g(X, W)+\alpha(X, Z) g(Y, W)- \\
& -\alpha(X, W) g(Y, Z)+\alpha(Y, W) g(X, Z)
\end{aligned}
$$

for any vector fields $X, Y, Z, W$ on $N$ [19], where $(0,2)$-tensor field $\alpha$ is given by

$$
\alpha(X, Y)=(\stackrel{\circ}{\widetilde{\nabla} w}) Y-w(X) w(Y)+\frac{1}{2} w(U) g(X, Y)
$$

for $X, Y \in \chi(M)$, where the trace of $\alpha$ is denoted by

$$
\operatorname{trace} \alpha=\lambda \text {. }
$$

Denote by $K(\pi)$ or $K(u, v)$ the sectional curvature of $M$ associated with a 2-plane section $\pi \subset T_{x} M$ with respect to the induced semi-symmetric non-metric connection $\nabla$, where $\{u, v\}$ is an orthonormal basis of $\pi$. The scalar curvature $\tau$ at $x \in M$ is denoted by

$$
\tau(x)=\sum_{1 \leq i<j \leq n} K\left(e_{i} \wedge e_{j}\right),
$$

where $\left\{e_{1}, \ldots, e_{n}\right\}$ is any orthonormal basis of $T_{x} M[8]$.

We will need the following Chen's lemma for later use:

2.1. Lemma. [6] Let $n \geq 2$ and $a_{1}, a_{2}, \ldots, a_{n}, b$ be real numbers such that

$$
\left(\sum_{i=1}^{n} a_{i}\right)^{2}=(n-1)\left(\sum_{i=1}^{n} a_{i}^{2}+b\right) .
$$

Then $2 a_{1} a_{2} \geq b$, with equality holding if and only if

$$
a_{1}+a_{2}=a_{3}=\ldots=a_{n}
$$


Let $M$ be an $n$-dimensional Riemannian manifold, $L$ a $k$-plane section of $T_{x} M, x \in M$ and $X$ a unit vector in $L$.

For an orthonormal basis $\left\{e_{1}, \ldots, e_{k}\right\}$ of $L$ such that $e_{1}=X$, the Ricci curvature (or $k$-Ricci curvature) of $L$ at $X$ is defined by

$$
\operatorname{Ric}_{L}(X)=K_{12}+K_{13}+\ldots+K_{1 k},
$$

where $K_{i j}$ denotes the sectional curvature of the 2-plane section spanned by $e_{i}$ and $e_{j}$. For any integer $k, 2 \leq k \leq n$, the Riemannian invariant $\Theta_{k}$ of $M$ is denoted by

$$
\Theta_{k}(x)=\frac{1}{k-1} \inf _{L, X} \operatorname{Ric}_{L}(X), \quad x \in M,
$$

where $L$ runs over all $k$-plane sections in $T_{x} M$ and $X$ runs over all unit vectors in $L$.

\section{Chen inequality for submanifolds of generalized complex space forms}

We consider as an ambient space a generalized complex space form with a semisymmetric metric connection.

A $2 m$-dimensional almost Hermitian manifold $(N, J, g)$ is said to be a generalized complex space form (see [17] and [18]) if there exist two functions $F_{1}$ and $F_{2}$ on $N$ such that

$$
\begin{aligned}
& \stackrel{\circ}{\widetilde{R}}(X, Y, Z, W)=F_{1}[g(Y, Z) g(X, W)-g(X, Z) g(Y, W)]+ \\
& +F_{2}[g(X, J Z) g(J Y, W)-g(Y, J Z) g(J X, W)+2 g(X, J Y) g(J Z, W)],
\end{aligned}
$$

for any vector fields $X, Y, Z, W$ on $N$, where $\stackrel{\circ}{\widetilde{R}}$ is the curvature tensor of $N$ with respect to the Levi-Civita connection $\stackrel{\circ}{\widetilde{\nabla}}$. In such a case, we will write $N\left(F_{1}, F_{2}\right)$.

If $N\left(F_{1}, F_{2}\right)$ is a generalized complex space form with a semi-symmetric metric connection $\widetilde{\nabla}$, then by the use of (2.6) and (3.1), the curvature tensor $\widetilde{R}$ of $N\left(F_{1}, F_{2}\right)$ can be written as

$$
\begin{aligned}
& \widetilde{R}(X, Y, Z, W)=F_{1}[g(Y, Z) g(X, W)-g(X, Z) g(Y, W)]+ \\
& +F_{2}[g(X, J Z) g(J Y, W)-g(Y, J Z) g(J X, W)+2 g(X, J Y) g(J Z, W)]- \\
& -\alpha(Y, Z) g(X, W)+\alpha(X, Z) g(Y, W)-\alpha(X, W) g(Y, Z)+\alpha(Y, W) g(X, Z) .
\end{aligned}
$$

Let $M$ be an $n$-dimensional, $n \geq 3$, submanifold of a $2 m$-dimensional generalized complex space form $N\left(F_{1}, F_{2}\right)$. We put

$$
J X=P X+F X,
$$

for any vector field $X$ tangent to $M$, where $P X$ and $F X$ are tangential and normal components of $J X$, respectively.

We also set

$$
\|P\|^{2}=\sum_{i, j=1}^{n} g^{2}\left(J e_{i}, e_{j}\right) .
$$


On the other hand, $\Theta^{2}(\pi)$ is denoted by $\Theta^{2}(\pi)=g^{2}\left(P e_{1}, e_{2}\right)=g^{2}\left(J e_{1}, e_{2}\right)$ in [2], where $\left\{e_{1}, e_{2}\right\}$ is an orthonormal basis of a 2-plane section $\pi . \Theta^{2}(\pi)$ is a real number in $[0,1]$, independent of the choice of $e_{1}$ and $e_{2}$.

For submanifolds of generalized complex space forms with respect to the semi-symmetric metric connection we establish the following sharp inequality:

3.1. Theorem. Let $M, n \geq 3$, be an $n$-dimensional submanifold of a $2 m$-dimensional generalized complex space form $N\left(F_{1}, F_{2}\right)$ with respect to the semi-symmetric metric connection $\widetilde{\nabla}$. Then we have:

$$
\begin{aligned}
\tau(x)-K(\pi) \leq & \frac{n-2}{2}\left[\frac{n^{2}}{n-1}\|H\|^{2}+(n+1) F_{1}-2 \lambda\right]- \\
& -\left[6 \Theta^{2}(\pi)-3\|P\|^{2}\right] \frac{F_{2}}{2}-\operatorname{trace}\left(\alpha_{\mid \pi \perp}\right),
\end{aligned}
$$

where $\pi$ is a 2 -plane section of $T_{x} M, x \in M$.

Proof. Let $\left\{e_{1}, e_{2}, \ldots e_{n}\right\}$ be an orthonormal basis of $T_{x} M$ and $\left\{e_{n+1}, \ldots, e_{2 m}\right\}$ be an orthonormal basis of $T_{x}^{\perp} M, x \in M$, where $e_{n+1}$ is parallel to the mean curvature vector $H$.

Taking $X=W=e_{i}$ and $Y=Z=e_{j}$ such that $i \neq j$ and by the use of (3.2), we get

$$
\widetilde{R}\left(e_{i}, e_{j}, e_{j}, e_{i}\right)=F_{1}+3 F_{2} g^{2}\left(J e_{i}, e_{j}\right)-\alpha\left(e_{i}, e_{i}\right)-\alpha\left(e_{j}, e_{j}\right) .
$$

From [16], the Gauss equation with respect to the semi-symmetric metric connection can be written as

$$
\widetilde{R}\left(e_{i}, e_{j}, e_{j}, e_{i}\right)=R\left(e_{i}, e_{j}, e_{j}, e_{i}\right)+g\left(\sigma\left(e_{i}, e_{j}\right), \sigma\left(e_{i}, e_{j}\right)\right)-g\left(\sigma\left(e_{i}, e_{i}\right), \sigma\left(e_{j}, e_{j}\right)\right) .
$$

Comparing the right hand sides of the equations (3.4) and (3.5), we obtain

$$
\begin{aligned}
& F_{1}+3 F_{2} g^{2}\left(J e_{i}, e_{j}\right)-\alpha\left(e_{i}, e_{i}\right)-\alpha\left(e_{j}, e_{j}\right) \\
= & R\left(e_{i}, e_{j}, e_{j}, e_{i}\right)+g\left(\sigma\left(e_{i}, e_{j}\right), \sigma\left(e_{i}, e_{j}\right)\right)-g\left(\sigma\left(e_{i}, e_{i}\right), \sigma\left(e_{j}, e_{j}\right)\right) .
\end{aligned}
$$

Then, by summation over $1 \leq i, j \leq n$, the above equation turns into

$$
\begin{aligned}
& 2 \tau+\|\sigma\|^{2}-n^{2}\|H\|^{2} \\
= & n(n-1) F_{1}+3 F_{2} \sum_{i, j=1}^{n} g^{2}\left(J e_{i}, e_{j}\right)-2(n-1) \lambda,
\end{aligned}
$$

where

$$
\|\sigma\|^{2}=\sum_{i, j=1}^{n} g\left(\sigma\left(e_{i}, e_{j}\right), \sigma\left(e_{i}, e_{j}\right)\right)
$$

and

$$
H=\frac{1}{n} \operatorname{trace} \sigma .
$$

We set

$$
\delta=2 \tau-\frac{n^{2}(n-2)}{n-1}\|H\|^{2}+2(n-1) \lambda-n(n-1) F_{1}-3 F_{2}\|P\|^{2} .
$$


Then, the equation (3.6) can be written as follows

$$
n^{2}\|H\|^{2}=(n-1)\left(\|\sigma\|^{2}+\delta\right) \text {. }
$$

For a chosen orthonormal basis, the relation (3.8) takes the following form

$$
\left(\sum_{i=1}^{n} \sigma_{i i}^{n+1}\right)^{2}=(n-1)\left[\sum_{i=1}^{n}\left(\sigma_{i i}^{n+1}\right)^{2}+\sum_{i \neq j}\left(\sigma_{i j}^{n+1}\right)^{2}+\sum_{r=n+2}^{2 m} \sum_{i, j=1}^{n}\left(\sigma_{i j}^{r}\right)^{2}+\delta\right] .
$$

So, by the use of Chen's Lemma, we have

$$
2 \sigma_{11}^{n+1} \sigma_{22}^{n+1}=\sum_{1 \leq i \neq j \leq n}\left(\sigma_{i j}^{n+1}\right)^{2}+\sum_{r=n+2}^{2 m} \sum_{i, j=1}^{n}\left(\sigma_{i j}^{r}\right)^{2}+\delta .
$$

Let $\pi$ be a 2-plane section of $T_{x} M$ at a point $x$, where $\pi=s p\left\{e_{1}, e_{2}\right\}$. Then, the Gauss equation for $X=Z=e_{1}$ and $Y=W=e_{2}$ gives us

$$
\begin{aligned}
& K(\pi)=F_{1}+3 F_{2} g^{2}\left(J e_{1}, e_{2}\right)-\alpha\left(e_{1}, e_{1}\right)-\alpha\left(e_{2}, e_{2}\right)+\sum_{r=n+1}^{2 m}\left[\sigma_{11}^{r} \sigma_{22}^{r}-\left(\sigma_{12}^{r}\right)^{2}\right] \geq \\
& \geq F_{1}+3 F_{2} g^{2}\left(J e_{1}, e_{2}\right)-\alpha\left(e_{1}, e_{1}\right)-\alpha\left(e_{2}, e_{2}\right)+ \\
& +\frac{1}{2}\left(\sum_{1 \leq i \neq j \leq n}\left(\sigma_{i j}^{n+1}\right)^{2}+\sum_{r=n+2}^{2 m} \sum_{i, j=1}^{n}\left(\sigma_{i j}^{r}\right)^{2}+\delta\right)+\sum_{r=n+2}^{2 m} \sigma_{11}^{r} \sigma_{22}^{r}-\sum_{r=n+1}^{2 m}\left(\sigma_{12}^{r}\right)^{2} \\
& =F_{1}+3 F_{2} g^{2}\left(J e_{1}, e_{2}\right)-\alpha\left(e_{1}, e_{1}\right)-\alpha\left(e_{2}, e_{2}\right)+ \\
& +\frac{1}{2} \sum_{1 \leq i \neq j \leq n}\left(\sigma_{i j}^{n+1}\right)^{2}+\frac{1}{2} \sum_{r=n+2}^{2 m} \sum_{i, j>2}^{n}\left(\sigma_{i j}^{r}\right)^{2}+ \\
& +\frac{1}{2} \sum_{r=n+2}^{2 m}\left(\sigma_{11}^{r}+\sigma_{22}^{r}\right)^{2}+\sum_{j>2}\left[\left(\sigma_{1 j}^{n+1}\right)^{2}+\left(\sigma_{2 j}^{n+1}\right)^{2}\right]+\frac{1}{2} \delta \geq \\
& \geq F_{1}+3 F_{2} g^{2}\left(J e_{1}, e_{2}\right)-\alpha\left(e_{1}, e_{1}\right)-\alpha\left(e_{2}, e_{2}\right)+\frac{1}{2} \delta
\end{aligned}
$$

which implies

$$
K(\pi) \geq F_{1}+3 F_{2} g^{2}\left(J e_{1}, e_{2}\right)-\alpha\left(e_{1}, e_{1}\right)-\alpha\left(e_{2}, e_{2}\right)+\frac{1}{2} \delta .
$$

From (3.7), it is easy to see that

$$
\begin{aligned}
K(\pi) \geq & \tau-\frac{n-2}{2}\left[\frac{n^{2}}{n-1}\|H\|^{2}+(n+1) F_{1}-2 \lambda\right]+ \\
& +\left[6 \Theta^{2}(\pi)-3\|P\|^{2}\right] \frac{F_{2}}{2}+\operatorname{trace}\left(\alpha_{\mid \pi^{\perp}}\right)
\end{aligned}
$$

where $\operatorname{trace}\left(\alpha_{\mid \pi^{\perp}}\right)$ is denoted by

$$
\alpha\left(e_{1}, e_{1}\right)+\alpha\left(e_{2}, e_{2}\right)=\lambda-\operatorname{trace}\left(\alpha_{\mid \pi^{\perp}}\right)
$$

(see [15]). Hence, we finish the proof of the theorem.

3.2. Proposition. The mean curvature $H$ of $M$ admitting semi-symmetric metric connection coincides with the mean curvature $\stackrel{\circ}{H}$ of $M$ admitting Levi-Civita connection if and only if the vector field $U$ is tangent to $M$.

As a consequence of Proposition 3.2 we can give the following result: 
3.3. Theorem. If the vector field $U$ is tangent to $M$, then the equality case of (3.3) holds at a point $x \in M$ if and only if there exist an orthonormal basis $\left\{e_{1}, e_{2}, \ldots e_{n}\right\}$ of $T_{x} M$ and an orthonormal basis $\left\{e_{n+1}, \ldots, e_{2 m}\right\}$ of $T_{x}^{\perp} M$ such that the shape operators of $M$ in $N\left(F_{1}, F_{2}\right)$ at $x$ have the following forms:

$$
A_{e_{n+1}}=\left(\begin{array}{ccccc}
a & 0 & 0 & \cdots & 0 \\
0 & b & 0 & \cdots & 0 \\
0 & 0 & \mu & \cdots & 0 \\
\vdots & \vdots & \vdots & \ddots & \vdots \\
0 & 0 & 0 & \cdots & \mu
\end{array}\right), \quad a+b=\mu
$$

and

$$
A_{e_{r}}=\left(\begin{array}{ccccc}
\sigma_{11}^{r} & \sigma_{12}^{r} & 0 & \cdots & 0 \\
\sigma_{12}^{r} & -\sigma_{11}^{r} & 0 & \cdots & 0 \\
0 & 0 & 0 & \cdots & 0 \\
\vdots & \vdots & \vdots & \ddots & \vdots \\
0 & 0 & 0 & \cdots & 0
\end{array}\right), \quad n+2 \leq i \leq 2 m
$$

where we denote by $\sigma_{i j}^{r}=g\left(\sigma\left(e_{i}, e_{j}\right), e_{r}\right), 1 \leq i, j \leq n$ and $n+2 \leq r \leq 2 m$.

Proof. Equality case holds at a point $x \in M$ if and only if the equality holds in each of the previous inequalities and hence the Lemma yields equality.

$$
\begin{gathered}
\sigma_{i j}^{n+1}=0, \quad \forall i \neq j, i, j>2, \\
\sigma_{i j}^{r}=0, \quad \forall i \neq j, i, j>2, r=n+1, \ldots, 2 m, \\
\sigma_{11}^{r}+\sigma_{22}^{r}=0, \quad \forall r=n+2, \ldots, 2 m, \\
\sigma_{1 j}^{n+1}=\sigma_{2 j}^{n+1}=0, \quad \forall j>2, \\
\sigma_{11}^{n+1}+\sigma_{22}^{n+1}=\sigma_{33}^{n+1}=\ldots=\sigma_{n n}^{n+1} .
\end{gathered}
$$

If we choose $\left\{e_{1}, e_{2}\right\}$ such that $\sigma_{12}^{n+1}=0$ and denote by $a=\sigma_{11}^{r}, b=\sigma_{22}^{r}, \mu=\sigma_{33}^{n+1}=$ $\ldots=\sigma_{n n}^{n+1}$, then the shape operators take the desired forms.

\section{Ricci curvature for submanifolds of generalized complex space forms}

In this section we establish relationship between the Ricci curvature of a submanifold $M$ in a generalized complex space form $N\left(F_{1}, F_{2}\right)$ with a semi-symmetric metric connection, and the squared mean curvature $\|H\|^{2}$.

Now, let begin with the following theorem:

4.1. Theorem. Let $M, n \geq 3$, be an $n$-dimensional submanifold of a $2 m$-dimensional generalized complex space form $N\left(F_{1}, F_{2}\right)$ with respect to the semi-symmetric metric connection $\widetilde{\nabla}$. Then we have:

$$
\|H\|^{2} \geq \frac{2 \tau}{n(n-1)}+\frac{2}{n} \lambda-F_{1}-\frac{3 F_{2}}{n(n-1)}\|P\|^{2} .
$$


Proof. Let $\left\{e_{1}, e_{2}, \ldots, e_{n}\right\}$ be an orthonormal basis of $T_{x} M$ and $\left\{e_{n+1}, \ldots, e_{2 m}\right\}$ be an orthonormal basis of $T_{x}^{\perp} M$ at $x \in M$, where $e_{n+1}$ is parallel to the mean curvature vector $H$.

Then, the equation (3.7) can be written as follows

$$
n^{2}\|H\|^{2}=2 \tau+\|\sigma\|^{2}+2(n-1) \lambda-n(n-1) F_{1}-3 F_{2}\|P\|^{2} .
$$

For a choosen orthonormal basis, let $e_{1}, e_{2}, \ldots, e_{n}$ diagonalize the shape operator $A_{e_{n+1}}$. Then, the shape operators take the forms

$$
A_{e_{n+1}}=\left(\begin{array}{cccc}
a_{1} & 0 & \cdots & 0 \\
0 & a_{2} & \cdots & 0 \\
\vdots & \vdots & \ddots & \vdots \\
0 & 0 & \cdots & a_{n}
\end{array}\right)
$$

and

$$
A_{e_{r}}=\left(\sigma_{i j}^{r}\right), \quad i, j=1, \ldots, n ; \quad r=n+2, \ldots, 2 m, \quad \operatorname{trace}_{e_{r}}=0 .
$$

By the use of (4.2), we obtain

$$
\begin{aligned}
n^{2}\|H\|^{2}= & 2 \tau+\sum_{i=1}^{n} a_{i}^{2}+\sum_{r=n+2}^{2 m} \sum_{i, j=1}^{n}\left(\sigma_{i j}^{r}\right)^{2}+ \\
& +2(n-1) \lambda-n(n-1) F_{1}-3 F_{2}\|P\|^{2} .
\end{aligned}
$$

On the other hand, since

$$
0 \leq \sum_{i<j}\left(a_{i}-a_{j}\right)^{2}=(n-1) \sum_{i=1}^{n} a_{i}^{2}-2 \sum_{i<j} a_{i} a_{j},
$$

we get

$$
n^{2}\|H\|^{2}=\left(\sum_{i=1}^{n} a_{i}\right)^{2}=\sum_{i=1}^{n} a_{i}^{2}+2 \sum_{i<j} a_{i} a_{j} \leq n \sum_{i=1}^{n} a_{i}^{2},
$$

which means

$$
\sum_{i=1}^{n} a_{i}^{2} \geq n\|H\|^{2}
$$

Thus, in view of (4.4) in (4.3) we get (4.1), which completes the proof of the theorem.

In view of Theorem 4.1, we can give the following theorem:

4.2. Theorem. Let $M, n \geq 3$, be an $n$-dimensional submanifold of a $2 m$-dimensional generalized complex space form $N\left(F_{1}, F_{2}\right)$ with respect to the semi-symmetric metric connection $\widetilde{\nabla}$ such that the vector field $U$ is tangent to $M$. Then, for any integer $k$, $2 \leq k \leq n$ and for any point $x \in M$, we have:

$$
\|H\|^{2}(x) \geq \Theta_{k}(\pi)+\frac{2}{n} \lambda-F_{1}-\frac{3 F_{2}}{n(n-1)}\|P\|^{2} .
$$


Proof. Let $\left\{e_{1}, e_{2}, \ldots, e_{n}\right\}$ be an orthonormal basis of $T_{x} M$ at $x \in M$. The $k$-plane section spanned by $e_{i_{1}}, \ldots, e_{i_{k}}$ is denoted by $L_{i_{1} \ldots i_{k}}$. Then, by the definitions, we can write

$$
\tau\left(L_{i_{1} \ldots i_{k}}\right)=\frac{1}{2} \sum_{i \in\left\{i_{1} \ldots i_{k}\right\}} \operatorname{Ric}_{L_{i_{1} \ldots i_{k}}}\left(e_{i}\right)
$$

and

$$
\tau(x)=\frac{1}{C_{n-2}^{k-2}} \sum_{1 \leq i_{1} \leq \ldots \leq i_{k} \leq n} \tau\left(L_{i_{1} \ldots i_{k}}\right) .
$$

By making use of (4.6) and (4.7) in (4.1), we obtain

$$
\tau(x) \geq \frac{n(n-1)}{2} \Theta_{k}(\pi)
$$

which gives us (4.5).

\section{Chen inequality for submanifolds of generalized Sasakian space forms}

Let $N$ be a $(2 m+1)$-dimensional almost contact metric manifold [5] with an almost contact metric structure $(\varphi, \xi, \eta, g)$ consisting of a $(1,1)$-tensor field $\varphi$, a vector field $\xi$, a 1-form $\eta$ and a Riemannian metric $g$ on $N$ satisfying

$$
\begin{aligned}
& \varphi^{2} X=-X+\eta(X) \xi, \quad \eta(\xi)=1, \quad \varphi \xi=0, \quad \eta \circ \varphi=0, \\
& g(\varphi X, \varphi Y)=g(X, Y)-\eta(X) \eta(Y), \quad g(X, \xi)=\eta(X),
\end{aligned}
$$

for all vector fields $X, Y$ on $N$. Such a manifold is said to be a contact metric manifold if $d \eta=\Phi$, where $\Phi(X, Y)=g(X, \varphi Y)$ is called the fundamental 2-form of $N$ [5].

On the other hand, the almost contact metric structure of $N$ is said to be normal if

$$
[\varphi, \varphi](X, Y)=-2 d \eta(X, Y) \xi
$$

for any vector fields $X, Y$ on $N$, where $[\varphi, \varphi]$ denotes the Nijenhuis torsion of $\varphi$, given by

$$
[\varphi, \varphi](X, Y)=\varphi^{2}[X, Y]+[\varphi X, \varphi Y]-\varphi[\varphi X, Y]-\varphi[X, \varphi Y]
$$

A normal contact metric manifold is called a Sasakian manifold [5].

Given an almost contact metric manifold $N$ with an almost contact metric structure $(\varphi, \xi, \eta, g), N$ is called a generalized Sasakian space form [1] if there exist three functions $f_{1}, f_{2}$ and $f_{3}$ on $N$ such that

$$
\begin{aligned}
& \stackrel{\circ}{\widetilde{R}}(X, Y, Z, W)=f_{1}\{g(Y, Z) g(X, W)-g(X, Z) g(Y, W)\}+ \\
& +f_{2}\{g(X, \varphi Z) g(\varphi Y, W)-g(Y, \varphi Z) g(\varphi X, W)+2 g(X, \varphi Y) g(\varphi Z, W)\}+ \\
& +f_{3}\{\eta(X) \eta(Z) g(Y, W)-\eta(Y) \eta(Z) g(X, W)+\eta(Y) \eta(W) g(X, Z)-\eta(X) \eta(W) g(Y, Z)\},
\end{aligned}
$$

for any vector fields $X, Y, Z, W$ on $N$, where $\stackrel{\circ}{\widetilde{R}}$ denotes the curvature tensor of $N$ with respect to the Levi-Civita connection $\stackrel{\circ}{\widetilde{\nabla}}$. In such a case, we will write $N\left(f_{1}, f_{2}, f_{3}\right)$. If $f_{1}=\frac{c+3}{4}, f_{2}=f_{3}=\frac{c-1}{4}$, then $N$ is a Sasakian space form. 
If $N\left(f_{1}, f_{2}, f_{3}\right)$ is a $(2 m+1)$-dimensional generalized Sasakian space form with respect to the semi-symmetric metric connection $\widetilde{\nabla}$. Then, from (2.6) and (5.1) the curvature tensor $\widetilde{R}$ of $N\left(f_{1}, f_{2}, f_{3}\right)$ can be written as follows

$$
\begin{aligned}
& \widetilde{R}(X, Y, Z, W)=f_{1}\{g(Y, Z) g(X, W)-g(X, Z) g(Y, W)\}+ \\
& +f_{2}\{g(X, \varphi Z) g(\varphi Y, W)-g(Y, \varphi Z) g(\varphi X, W)+2 g(X, \varphi Y) g(\varphi Z, W)\}+ \\
& +f_{3}\{\eta(X) \eta(Z) g(Y, W)-\eta(Y) \eta(Z) g(X, W)+\eta(Y) \eta(W) g(X, Z)-\eta(X) \eta(W) g(Y, Z)\}- \\
& -\alpha(Y, Z) g(X, W)+\alpha(X, Z) g(Y, W)-\alpha(X, W) g(Y, Z)+\alpha(Y, W) g(X, Z) .
\end{aligned}
$$

Let $M, n \geq 3$, be an $n$-dimensional submanifold of a $(2 m+1)$-dimensional generalized Sasakian space form. We put

$$
\varphi X=P X+F X
$$

for any vector field $X$ tangent to $M$, where $P X$ and $F X$ are tangential and normal components of $\varphi X$, respectively.

We also set

$$
\|P\|^{2}=\sum_{i, j=1}^{n} g^{2}\left(\varphi e_{i}, e_{j}\right) .
$$

Decompose

$$
\xi=\xi^{\top}+\xi^{\perp},
$$

where $\xi^{\top}$ and $\xi^{\perp}$ denote the tangential and normal components of $\xi$.

From [2], recall $\Theta^{2}(\pi)=g^{2}\left(P e_{1}, e_{2}\right)=g^{2}\left(\varphi e_{1}, e_{2}\right)$, where $\left\{e_{1}, e_{2}\right\}$ is an orthonormal basis of a 2-plane section $\pi$, is a real number in $[0,1]$, independent of the choice of $e_{1}$ and $e_{2}$.

Now, let begin with the following theorem which gives us a sharp inequality for submanifolds of generalized Sasakian space forms with respect to the semi-symmetric metric connection:

5.1. Theorem. Let $M, n \geq 3$, be an $n$-dimensional submanifold of a $(2 m+1)$-dimensional generalized Sasakian space form $N\left(f_{1}, f_{2}, f_{3}\right)$ with respect to the semi-symmetric metric connection $\widetilde{\nabla}$. Then we have:

$$
\begin{aligned}
\tau(x)-K(\pi) \leq & (n-2)\left[\frac{n^{2}}{2(n-1)}\|H\|^{2}+(n+1) \frac{f_{1}}{2}-\lambda\right]- \\
& -\left[6 \Theta^{2}(\pi)-3\|P\|^{2}\right] \frac{f_{2}}{2}+\left[\left\|\xi_{\pi}\right\|^{2}-(n-1)\left\|\xi^{\top}\right\|^{2}\right] f_{3}- \\
& -\operatorname{trace}\left(\alpha_{\mid \pi^{\perp}}\right),
\end{aligned}
$$

where $\pi$ is a 2 -plane section of $T_{x} M, x \in M$.

Proof. Let $\left\{e_{1}, e_{2}, \ldots e_{n}\right\}$ be an orthonormal basis of $T_{x} M$ and $\left\{e_{n+1}, \ldots, e_{2 m+1}\right\}$ be an orthonormal basis of $T_{x}^{\perp} M, x \in M$, where $e_{n+1}$ is parallel to the mean curvature vector $H$. 
For $X=W=e_{i}$ and $Y=Z=e_{j}$ such that $i \neq j$, the equation (5.2) can be written as

$$
\widetilde{R}\left(e_{i}, e_{j}, e_{j}, e_{i}\right)=f_{1}+3 f_{2} g^{2}\left(\varphi e_{i}, e_{j}\right)-f_{3}\left[\eta\left(e_{i}\right)^{2}+\eta\left(e_{j}\right)^{2}\right]-\alpha\left(e_{1}, e_{1}\right)-\alpha\left(e_{2}, e_{2}\right) .
$$

Comparing the right hand sides of the equations (3.5) and (5.4) we can write

$$
\begin{aligned}
& f_{1}+3 f_{2} g^{2}\left(\varphi e_{i}, e_{j}\right)-f_{3}\left[\eta\left(e_{i}\right)^{2}+\eta\left(e_{j}\right)^{2}\right]-\alpha\left(e_{1}, e_{1}\right)-\alpha\left(e_{2}, e_{2}\right) \\
= & R\left(e_{i}, e_{j}, e_{j}, e_{i}\right)+g\left(\sigma\left(e_{i}, e_{j}\right), \sigma\left(e_{i}, e_{j}\right)\right)-g\left(\sigma\left(e_{i}, e_{i}\right), \sigma\left(e_{j}, e_{j}\right)\right) .
\end{aligned}
$$

Then, by summation over $1 \leq i, j \leq n$, the above relation reduces to

$$
2 \tau+\|\sigma\|^{2}-n^{2}\|H\|^{2}=n(n-1) f_{1}+3 f_{2}\|P\|^{2}-2(n-1) f_{3}\left\|\xi^{\top}\right\|^{2}-2(n-1) \lambda .
$$

If we put

$$
\delta=2 \tau-\frac{n^{2}(n-2)}{n-1}\|H\|^{2}+2(n-1) \lambda-n(n-1) f_{1}-3 f_{2}\|P\|^{2}+2(n-1) f_{3}\left\|\xi^{\top}\right\|^{2},
$$

the equation (5.5) turns into

$$
n^{2}\|H\|^{2}=(n-1)\left(\|\sigma\|^{2}+\delta\right) \text {. }
$$

For a chosen orthonormal basis, the relation (5.7) takes the following form

$$
\left(\sum_{i=1}^{n} \sigma_{i i}^{n+1}\right)^{2}=(n-1)\left[\sum_{i=1}^{n}\left(\sigma_{i i}^{n+1}\right)^{2}+\sum_{i \neq j}\left(\sigma_{i j}^{n+1}\right)^{2}+\sum_{r=n+2}^{2 m+1} \sum_{i, j=1}^{n}\left(\sigma_{i j}^{r}\right)^{2}+\delta\right] .
$$

So, by the use of Chen's Lemma, we have

$$
2 \sigma_{11}^{n+1} \sigma_{22}^{n+1}=\sum_{1 \leq i \neq j \leq n}\left(\sigma_{i j}^{n+1}\right)^{2}+\sum_{r=n+2}^{2 m+1} \sum_{i, j=1}^{n}\left(\sigma_{i j}^{r}\right)^{2}+\delta
$$

Let $\pi$ be a 2-plane section of $T_{x} M$ at a point $x$, where $\pi=\operatorname{sp}\left\{e_{1}, e_{2}\right\}$. We need to denote $\xi_{\pi}=p r_{\pi} \xi$ for the later use as follows

$$
\left\|\xi_{\pi}\right\|^{2}=\eta\left(e_{1}\right)^{2}+\eta\left(e_{2}\right)^{2} .
$$

Then, from the Gauss equation for $X=Z=e_{1}$ and $Y=W=e_{2}$ we get

$$
\begin{aligned}
& K(\pi)=f_{1}+3 f_{2} g^{2}\left(P e_{1}, e_{2}\right)-f_{3}\left\|\xi_{\pi}\right\|^{2}-\alpha\left(e_{1}, e_{1}\right)-\alpha\left(e_{2}, e_{2}\right)+\sum_{r=n+1}^{2 m+1}\left[\sigma_{11}^{r} \sigma_{22}^{r}-\left(\sigma_{12}^{r}\right)^{2}\right] \geq \\
& \geq f_{1}+3 f_{2} g^{2}\left(P e_{1}, e_{2}\right)-f_{3}\left\|\xi_{\pi}\right\|^{2}-\alpha\left(e_{1}, e_{1}\right)-\alpha\left(e_{2}, e_{2}\right)+ \\
& \left.+\frac{1}{2} \sum_{1 \leq i \neq j \leq n}\left(\sigma_{i j}^{n+1}\right)^{2}+\sum_{r=n+2}^{2 m+1} \sum_{i, j=1}^{n}\left(\sigma_{i j}^{r}\right)^{2}+\delta\right)+\sum_{r=n+2}^{2 m+1} \sigma_{11}^{r} \sigma_{22}^{r}-\sum_{r=n+1}^{2 m+1}\left(\sigma_{12}^{r}\right)^{2} \\
& =f_{1}+3 f_{2} g^{2}\left(P e_{1}, e_{2}\right)-f_{3}\left\|\xi_{\pi}\right\|^{2}-\alpha\left(e_{1}, e_{1}\right)-\alpha\left(e_{2}, e_{2}\right)+ \\
& +\frac{1}{2} \sum_{1 \leq i \neq j \leq n}\left(\sigma_{i j}^{n+1}\right)^{2}+\frac{1}{2} \sum_{r=n+2}^{2 m+1} \sum_{i, j>2}^{n}\left(\sigma_{i j}^{r}\right)^{2}+ \\
& +\frac{1}{2} \sum_{r=n+2}^{2 m+1}\left(\sigma_{11}^{r}+\sigma_{22}^{r}\right)^{2}+\sum_{j>2}\left[\left(\sigma_{1 j}^{n+1}\right)^{2}+\left(\sigma_{2 j}^{n+1}\right)^{2}\right]+\frac{1}{2} \delta \geq \\
& \geq f_{1}+3 f_{2} g^{2}\left(P e_{1}, e_{2}\right)-f_{3}\left\|\xi_{\pi}\right\|^{2}-\alpha\left(e_{1}, e_{1}\right)-\alpha\left(e_{2}, e_{2}\right)+\frac{1}{2} \delta,
\end{aligned}
$$


which implies

$$
K(\pi) \geq f_{1}+3 f_{2} g^{2}\left(P e_{1}, e_{2}\right)-f_{3}\left\|\xi_{\pi}\right\|^{2}-\alpha\left(e_{1}, e_{1}\right)-\alpha\left(e_{2}, e_{2}\right)+\frac{1}{2} \delta .
$$

From (5.6), it easy to see that

$$
\begin{aligned}
K(\pi) \geq & \tau-(n-2)\left[\frac{n^{2}}{2(n-1)}\|H\|^{2}+(n+1) \frac{f_{1}}{2}-\lambda\right]- \\
& -\left[6 \Theta^{2}(\pi)-3\|P\|^{2}\right] \frac{f_{2}}{2}-\left[\left\|\xi_{\pi}\right\|^{2}-(n-1)\left\|\xi^{\top}\right\|^{2}\right] f_{3}+ \\
& +\operatorname{trace}\left(\alpha_{\mid \pi^{\perp}}\right),
\end{aligned}
$$

which gives us (5.3). Hence, we complete the proof of the theorem.

5.2. Corollary. Let $M, n \geq 3$, be an $n$-dimensional submanifold of a $(2 m+1)$ dimensional generalized Sasakian space form $N\left(f_{1}, f_{2}, f_{3}\right)$ with respect to the semisymmetric metric connection $\widetilde{\nabla}$.

If the structure vector field $\xi$ is tangent to $M$, we have

$$
\begin{aligned}
\tau(x)-K(\pi) \leq & (n-2)\left[\frac{n^{2}}{2(n-1)}\|H\|^{2}+(n+1) \frac{f_{1}}{2}-\lambda\right]- \\
& -\left[6 \Theta^{2}(\pi)-3\|P\|^{2}\right] \frac{f_{2}}{2}+\left[\left\|\xi_{\pi}\right\|^{2}-(n-1)\right] f_{3}- \\
& -\operatorname{trace}\left(\alpha_{\mid \pi^{\perp}}\right) .
\end{aligned}
$$

If the structure vector field $\xi$ is normal to $M$, we have

$$
\begin{aligned}
\tau(x)-K(\pi) \leq & (n-2)\left[\frac{n^{2}}{2(n-1)}\|H\|^{2}+(n+1) \frac{f_{1}}{2}-\lambda\right]- \\
& -\left[6 \Theta^{2}(\pi)-3\|P\|^{2}\right] \frac{f_{2}}{2}-\operatorname{trace}\left(\alpha_{\mid \pi^{\perp}}\right) .
\end{aligned}
$$

As a consequence of Proposition 3.2, for both submanifolds of generalized Sasakian space forms, we can give the following corollary:

5.3. Corollary. Under the same assumptions as in the Theorem 5.1, if the vector field $U$ is tangent to $M$, then we have:

$$
\begin{aligned}
\tau(x)-K(\pi) \leq & (n-2)\left[\frac{n^{2}}{2(n-1)}\|\stackrel{\circ}{H}\|^{2}+(n+1) \frac{f_{1}}{2}-\lambda\right]- \\
& -\left[6 \Theta^{2}(\pi)-3\|P\|^{2}\right] \frac{f_{2}}{2}+\left[\left\|\xi_{\pi}\right\|^{2}-(n-1)\right] f_{3}- \\
& -\operatorname{trace}\left(\alpha_{\mid \pi^{\perp}}\right) .
\end{aligned}
$$

5.4. Theorem. The equality case of (5.3) holds at a point $x \in M$ if and only if there exist an orthonormal basis $\left\{e_{1}, e_{2}, \ldots e_{n}\right\}$ of $T_{x} M$ and an orthonormal basis $\left\{e_{n+1}, \ldots, e_{2 m+1}\right\}$ of $T_{x}^{\perp} M$ such that the shape operators of $M$ in $N\left(f_{1}, f_{2}, f_{3}\right)$ at $x$ have the following forms:

$$
A_{e_{n+1}}=\left(\begin{array}{ccccc}
a & 0 & 0 & \cdots & 0 \\
0 & b & 0 & \cdots & 0 \\
0 & 0 & \mu & \cdots & 0 \\
\vdots & \vdots & \vdots & \ddots & \vdots \\
0 & 0 & 0 & \cdots & \mu
\end{array}\right), \quad a+b=\mu
$$


and

$$
A_{e_{r}}=\left(\begin{array}{ccccc}
\sigma_{11}^{r} & \sigma_{12}^{r} & 0 & \cdots & 0 \\
\sigma_{12}^{r} & -\sigma_{11}^{r} & 0 & \cdots & 0 \\
0 & 0 & 0 & \cdots & 0 \\
\vdots & \vdots & \vdots & \ddots & \vdots \\
0 & 0 & 0 & \cdots & 0
\end{array}\right), \quad n+2 \leq i \leq 2 m+1
$$

where we denote by $\sigma_{i j}^{r}=g\left(\sigma\left(e_{i}, e_{j}\right), e_{r}\right), 1 \leq i, j \leq n$ and $n+2 \leq r \leq 2 m+1$.

Proof. Equality case holds at a point $x \in M$ if and only if the equality holds in each of the previous inequalities and hence the Lemma yields equality.

$$
\begin{aligned}
& \sigma_{i j}^{n+1}=0, \quad \forall i \neq j, i, j>2, \\
& \sigma_{i j}^{r}=0, \quad \forall i \neq j, i, j>2, r=n+1, \ldots, 2 m+1, \\
& \sigma_{11}^{r}+\sigma_{22}^{r}=0, \quad \forall r=n+2, \ldots, 2 m+1, \\
& \sigma_{1 j}^{n+1}=\sigma_{2 j}^{n+1}=0, \quad \forall j>2, \\
& \sigma_{11}^{n+1}+\sigma_{22}^{n+1}=\sigma_{33}^{n+1}=\ldots=\sigma_{n n}^{n+1} .
\end{aligned}
$$

If we choose $\left\{e_{1}, e_{2}\right\}$ such that $\sigma_{12}^{n+1}=0$ and denote by $a=\sigma_{11}^{r}, b=\sigma_{22}^{r}, \mu=\sigma_{33}^{n+1}=$ $\ldots=\sigma_{n n}^{n+1}$, then the shape operators take the mentioned forms.

\section{Ricci curvature for submanifolds of generalized Sasakian space forms}

In this section we establish relationship between the Ricci curvature of a submanifold $M$ of a generalized Sasakian space form $N\left(f_{1}, f_{2}, f_{3}\right)$ with a semi-symmetric metric connection and the squared mean curvature $\|H\|^{2}$.

Now, let begin with the following theorem:

6.1. Theorem. Let $M, n \geq 3$, be an $n$-dimensional submanifold of a ( $2 m+1)$-dimensional generalized Sasakian space form $N\left(f_{1}, f_{2}, f_{3}\right)$ with respect to the semi-symmetric metric connection $\widetilde{\nabla}$. Then we have:

$$
\begin{aligned}
\|H\|^{2} \geq & \frac{2 \tau}{n(n-1)}+\frac{2}{n} \lambda-f_{1}-\frac{3 f_{2}}{n(n-1)}\|P\|^{2}+ \\
& +\frac{2}{n} f_{3}\left\|\xi^{\top}\right\|^{2} .
\end{aligned}
$$

Proof. Let $\left\{e_{1}, e_{2}, \ldots, e_{n}\right\}$ be an orthonormal basis of $T_{x} M$ and $\left\{e_{n+1}, \ldots, e_{2 m+1}\right\}$ be an orthonormal basis of $T_{x}^{\perp} M, x \in M$, where $e_{n+1}$ is parallel to the mean curvature vector $H$. Then, the equation (5.5) can be written as follows

$$
\begin{aligned}
n^{2}\|H\|^{2}= & 2 \tau+\|\sigma\|^{2}+2(n-1) \lambda-n(n-1) f_{1} \\
& -3 f_{2}\|P\|^{2}+2(n-1) f_{3} .
\end{aligned}
$$


For a choosen orthonormal basis, let $e_{1}, e_{2}, \ldots, e_{n}$ diagonalize the shape operator $A_{e_{n+1}}$. Then, the shape operators take the forms

$$
A_{e_{n+1}}=\left(\begin{array}{cccc}
a_{1} & 0 & \cdots & 0 \\
0 & a_{2} & \cdots & 0 \\
\vdots & \vdots & \ddots & \vdots \\
0 & 0 & \cdots & a_{n}
\end{array}\right)
$$

and

$$
A_{e_{r}}=\left(\sigma_{i j}^{r}\right), \quad i, j=1, \ldots, n ; \quad r=n+2, \ldots, 2 m+1, \quad \operatorname{trace} A_{e_{r}}=0 .
$$

By the use of (6.2), we obtain

$$
\begin{aligned}
n^{2}\|H\|^{2}= & 2 \tau+\sum_{i=1}^{n} a_{i}^{2}+\sum_{r=n+2}^{2 m+1} \sum_{i, j=1}^{n}\left(\sigma_{i j}^{r}\right)^{2}+ \\
& +2(n-1) \lambda-n(n-1) f_{1}-3 f_{2}\|P\|^{2}+2(n-1) f_{3} .
\end{aligned}
$$

On the other hand, we know that

$$
\sum_{i=1}^{n} a_{i}^{2} \geq n\|H\|^{2} .
$$

Hence, by the use of (6.4) in (6.3), we obtain (6.1).

In view of Theorem 6.1 , we can give the following theorem:

6.2. Theorem. Let $M, n \geq 3$, be an $n$-dimensional submanifold of a $(2 m+1)$-dimensional generalized Sasakian space form $N\left(f_{1}, f_{2}, f_{3}\right)$ with respect to the semi-symmetric metric connection $\widetilde{\nabla}$ such that the vector field $U$ is tangent to $M$. Then, for any integer $k$, $2 \leq k \leq n$ and for any point $x \in M$, we have:

$$
\|H\|^{2}(x) \geq \Theta_{k}(\pi)+\frac{2}{n} \lambda-f_{1}-\frac{3 f_{2}}{n(n-1)}\|P\|^{2}+\frac{2}{n} f_{3}\left\|\xi^{\top}\right\|^{2} .
$$

Proof. Similar to the proof of the Theorem 4.2, we easily get (6.5).

\section{References}

[1] Alegre, P., Blair, D. E., Carriazo, A.: Generalized Sasakian space forms, Israel J. of Math. 141, 157-183, (2004)

[2] Alegre, P., Carriazo A., Kim, Y. H., Yoon D. W.: B. Y. Chen's inequality for submanifolds of generalized space forms, Indian J. Pure Appl. Math. 38, 185-201, (2007)

[3] Arslan, K., Ezentaş, R., Mihai, I., Murathan, C., Özgür, C.: B. Y. Chen inequalities for submanifolds in locally conformal almost cosymplectic manifolds, Bull. Inst. Math. Acad. Sin. 29, 231-242, (2001)

[4] Arslan, K., Ezentaş, R., Mihai, I., Murathan, C., Özgür, C.: Certain inequalities for submanifolds in $(\kappa, \mu)$-contact space forms, Bull. Aust. Math. Soc. 64, 201-212, (2001)

[5] Blair, D. E.: Riemannian Geometry of Contact and Symplectic Manifolds, Birkhäuser, Boston, 2002.

[6] Chen, B. Y.: Some pinching and classification theorems for minimal submanifolds, Arch. Math. 60, 568-578, (1993) 
[7] Chen, B. Y.: Strings of Riemannian invariants, inequalities, ideal immersions and their applications, The Third Pacific Rim Geometry Conference (Seoul, 1996), 7-60, Monogr. Geom. Topology, 25, Int. Press, Cambridge, MA, 1998

[8] Chen, B. Y.: Some new obstructions to minimal and Lagrangian isometric immersions, Japanese J. Math. 26, 105-127, (2000)

[9] Chen, B. Y.: $\delta$-invariants, Inequalities of Submanifolds and Their Applications, in Topics in Differential Geometry, Eds. A. Mihai, I. Mihai, R. Miron, Editura Academiei Romane, Bucuresti, 29-156, (2008)

[10] A. Friedmann and J. A. Schouten, Über die Geometrie der halbsymmetrischen Übertragungen, (German) Math. Z. Vol. I. 21, 211-223, (1924)

[11] Hayden, H. A.: Subspace of a space with torsion, Proceedings of the London Mathematical Society II Series 34, 27-50, (1932)

[12] Imai, T.: Notes on semi-symmetric metric connections, Commemoration volumes for Prof. Dr. Akitsugu Kawaguchi's seventieth birthday, Vol. I. Tensor 24, 293-296, (1972)

[13] Imai, T.: Hypersurfaces of a Riemannian manifold with semi-symmetric metric connection, Tensor 23, 300-306 (1972).

[14] Mihai, A., Özgür, C.: Chen inequalities for submanifolds of real space forms with a semisymmetric metric connection, Taiwanese J. Math. 14, 1465-1477, (2010)

[15] Mihai, A., Özgür, C.: Chen inequalities for submanifolds of complex space forms and Sasakian space forms with semi-symmetric metric connections, Rocky Mountain J. Math. 41, 1653-1673, (2011)

[16] Nakao, Z.: Submanifolds of a Riemannian manifold with semi-symmetric metric connections, Proc. Amer. Math. Soc. 54, 261-266, (1976)

[17] Tricerri, F., Vanhecke, L.: Curvature tensors on almost Hermitian manifolds, Transactions of the American Mathematical Society 267, 365-398, (1981)

[18] Vanhecke, L.: Almost Hermitian manifolds with $J$-invariant Riemannian curvature tensor, Rendiconti del Seminario Mathematico della Universitá e Politecnico di Torino 34, 487-498, (1975)

[19] Yano, K.: On semi-symmetric metric connections, Rev. Roumaine Math. Pures Appl. 15, 1579-1586, (1970) 
